\title{
Results of Tailored CAS in Patients with High Risk Carotid Stenosis for CEA
}

Daisuke Wajima, Taiji Yonezawa, Masashi Kotsugi, and Shuta Aketa

Obejctive: This retrospective study aimed to compare the perioperative complications associated with treating carotid artery stenosis using either carotid endarterectomy (CEA) or carotid artery stenting (CAS) that were performed at a single Japanese institution from April 2011 to December 2014.

Methods: CEA or CAS was selected to treat 222 lesions based on the patients' individual risk factors. CEA was considered to be the first-line treatment in all cases $(n=104)$. The CEA group contained 55 symptomatic cases and 49 asymptomatic cases, and the CAS group contained 59 symptomatic cases and 59 asymptomatic cases. High-risk patients for CEA, which included, for example, those with angiographically poor collateral flows underwent CAS $(n=118)$.

Results: The CEA and CAS groups were compared with respect to the perioperative clinical complications that occurred within 30 days of the procedures, which included any strokes, myocardial infarctions, or deaths. No significant differences were observed between the groups in relation to the perioperative complications.

Conclusion: Selecting appropriate individualized treatment methods based on patients' risk factors may contribute to improvements in the overall outcomes in patients with carotid artery stenoses.

Keywords carotid endarterectomy (CEA), carotid artery stenting (CAS), carotid stenosis, CEA high-risk, perioperative complications

\section{Introduction}

Cervical carotid artery stenosis is a common cause of ipsilateral ischemic stroke. Preventive treatment for carotid artery stenosis includes medical and surgical treatments, for example, carotid endarterectomy (CEA) and carotid artery stenting (CAS).

To date, however, there is no established standardized criterion for the classification of treatment strategy for CEA/CAS patients as high-risk individuals. ${ }^{1-15)}$

Therefore, it is important to establish an optimal treatment strategy that is specific to carotid artery stenosis in Japan.

$\overline{\text { Department of Neurosurgery, Osaka Police Hospital, Osaka, }}$ Japan

Received: July 27, 2015; Accepted: November 17, 2015

Corresponding author: Daisuke Wajima, MD. Department of Neurosurgery, Osaka Police Hospital, Kitayama-cho 10 - 31, Tennoji, Osaka, 543-0035, Japan

Email:wajima@naramed-u.ac.jp

(C)2016 The Editorial Committee of Journal of Neuroendovascular Therapy. All rights reserved.
In recent years, the outcomes associated with CEA, CAS, and medical treatment for carotid artery stenosis have improved..$^{5,11-13)}$ However, no criteria have been developed to guide the classification of high-risk CEA and CAS patients. ${ }^{2,6,14-15)}$ In this study, we conducted a retrospective comparative investigation of all of the patients with carotid artery stenoses who were treated with CEA or CAS from April 2011 to December 2014 at our institution. We examined the CAS and CEA groups in relation to their perioperative complications.

\section{Methods}

\section{Case material}

We conducted a retrospective study of 211 consecutive patients (226 lesions) with carotid artery stenoses who underwent consecutive surgery. CEA and CAS were performed by the same operators (CEA was performed by T.Y and CAS was performed by S.A) at our hospital between April 2011 and December 2014. Our study population comprised patients with carotid artery stenoses who underwent either 
CAS or CEA ( $\geqq 50 \%$ over stenosis in symptomatic patients and $\geqq 60 \%$ stenosis in asymptomatic patients). ${ }^{11,13)}$ Before the patients underwent CEA or CAS, stress myocardial scintigraphy, coronary artery computed tomographic angiography (CTA), or coronary angiography were performed, as necessary in the Department of Cardiology. Thereafter, percutaneous coronary interventions (PCIs) were performed on 68 patients in CAS group and on none of the patients in the CEA group. The PCIs were followed by CEA or CAS.

\section{Carotid endarterectomy/carotid artery stenting risk classification}

High-risk CEA patients were defined as those in whom general anesthesia was considered to carry a high risk and in the current study they comprised those with severe pulmonary disease (6 cases: $5.1 \%),{ }^{6,15)}$ with left ventricular ejection fractions of $<30 \%$ ( 8 cases: $6.8 \%$ ), ${ }^{15-16)}$ with contralateral laryngeal nerve palsy ( 1 case: $1.2 \%){ }^{6}{ }^{6}$ who had undergone previous neck surgery ( 8 cases: $\left.7.2 \%),{ }^{6}\right)$ with a history of radiation to the neck (5 cases: $4.2 \%),{ }^{6}$ with lesions that were higher than the second cervical vertebra (39 cases: $32.6 \%$ ), ${ }^{6}$ with contralateral internal carotid artery (ICA) occlusions (34 cases: $28.4 \%$ ), ${ }^{6}$ and those who were being administered dual anti-platelet therapy following the PCI (13 cases: $11.5 \%)$. In addition, patients with poor collateral flows during the temporary occlusion of the ICA that is, during the MATAS test, were considered high-risk CEA patients (4 cases: $3.0 \%$ ), even though the authors use the intraluminalshunt device for selected cases in CEA.

\section{Criteria for the treatment selection}

Using the risk classification described above, CEA was performed on most of the low-risk patients $(n=104)$. CAS was performed on the high-risk CEA cases $(n=118)$.

\section{Perioperative complications}

The perioperative complications that were evaluated in this study were any strokes, myocardial infarction (MI)s, or deaths within 30 days of CEA or CAS. Stroke was defined as any relevant clinical feature that may have been caused by new ischemic events, specifically infarctions, transient ischemic attacks (TIAs), or intracerebral hemorrhages (ICHs). Minor strokes were defined as events that changed patients' modified Rankin Scales (mRS) to 0-1 points, and major strokes were defined as events that changed patients' $\mathrm{mRS}$ to $>2$ points. An MI was defined according to the symptoms, namely chest pain or discomfort, which were accompanied by an ST segment change on the electrocardiogram and-/or elevations in the enzyme levels. Post-CEA or CAS hyperperfusion syndrome was defined as the presence of the following clinical symptoms within 30 days of the procedures; evidence of hyperperfusion on transcranial Doppler, single photon emission computed tomography (CT) or CT perfusion or magnetic resonance (MR) perfusion imaging; clinical features that included the presence of new headaches, seizures, hemiparesis, or a score of $<15$ on the Glasgow Coma Scale (GCS) or radiological features that included cerebral edema or ICH; and no evidence of new cerebral ischemia on CT or magnetic resonance image (MRI) scans. The organization's institutional review board approved this study, and the requirement for full informed consent was obtained.

\section{Carotid endarterectomy procedure}

All patients were operated under general anesthesia. Routine monitoring of the patients during general anesthesia involved standard electrocardiography, the placement of a continuous intra-arterial blood pressure catheter to obtain direct arterial blood pressure measurements, pulse oximetry, and capnography. The local cerebral oxygenation was monitored bilaterally in all CEA cases using near infra- red spectroscopy on the bilateral frontal head portions. A bolus of heparin $(100 \mathrm{U} / \mathrm{kg})$ was injected before ICA clamping. We used an intraluminal shunt device for selected cases during plaque removal, if necessary. After plaque removal, the carotid artery was sutured with 6-0 nylon sutures. If necessary, tacking suturing was undertaken on the distal edge of the ICA. The systolic arterial blood pressure in all of the patients was maintained between $100 \mathrm{mmHg}$ and $140 \mathrm{mmHg}$ for 1 week postoperatively.

\section{Carotid artery stenting procedure}

Dual antiplatelet therapy that comprised aspirin $(100 \mathrm{mg} /$ day) and clopidogrel (75 mg/day) was initiated at least 1 week before CAS and statins were administered to all patients at least 2 weeks before CAS. CAS was performed on all patients under local anesthesia. Intraoperative heparinization was performed based on the activated clotting time, which was usually set to $>300 \mathrm{~s}$, or double the control values before the procedure began. A 9Fr OPTIMO balloon guiding catheter (Tokai Medical Products Inc. Kasugai, Aichi, Japan) was introduced just proximal to the cervical stenotic lesion via the right femoral artery (Fig. 1A). Distal protection was performed with Filter-Wire EZ (Boston Scientific, Natick, MA, USA) or Spider (ev3 Endovascular, Plymouth, MA, USA) and concomitant with proximal protection was performed with 9Fr OPTIMO balloon guiding catheter (Fig. 1B). Stent selection was based on the plaques' 


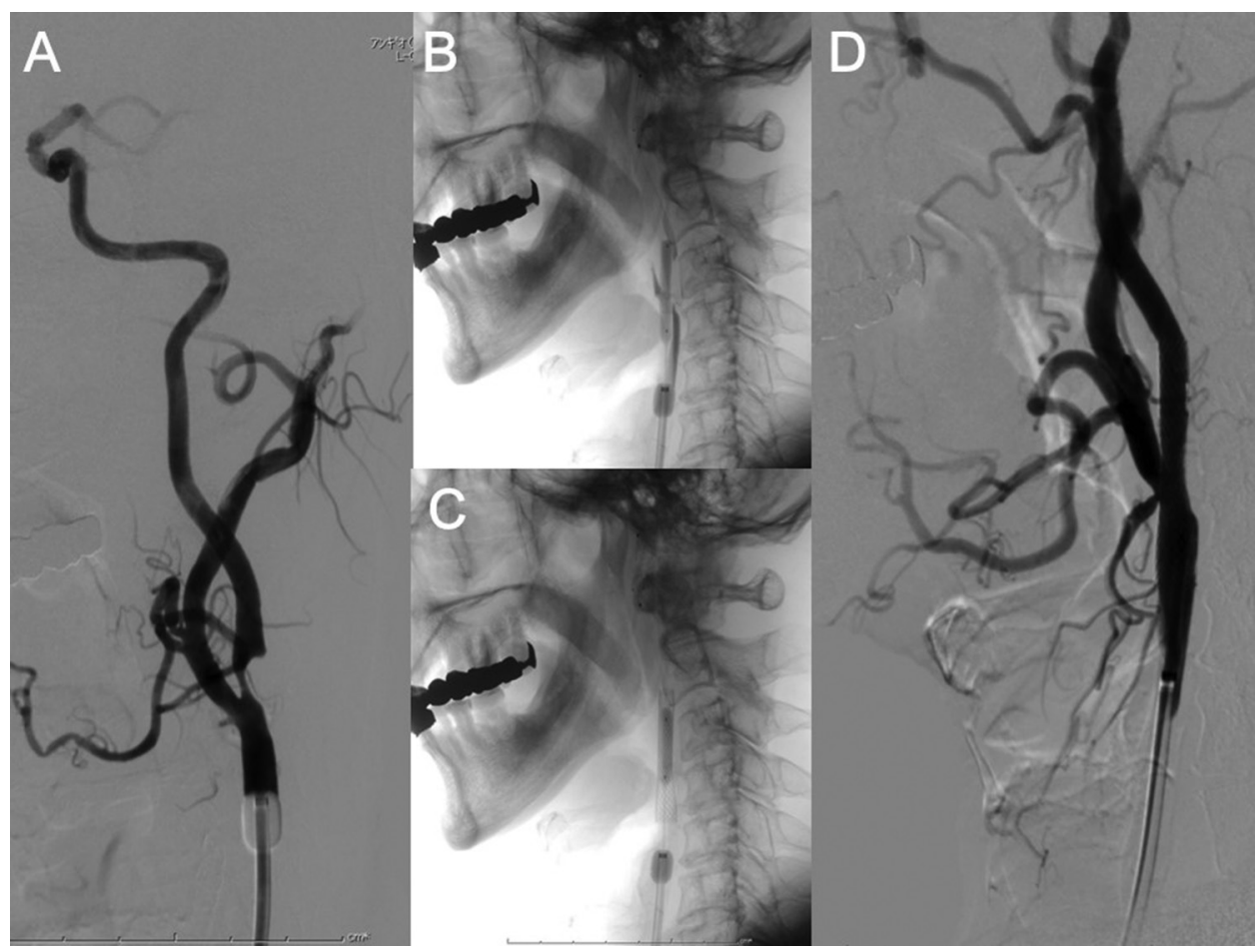

Fig. 1 The carotid artery stenting (CAS) procedure. Following proximal protection with a 9Fr OPTIMO balloon guiding catheter (A) and distal filter protection with a Filter-Wire EZ or Spider distal filter, (B) indicates pre-dilation was performed, (C) indicates post-dilation, DSA confirmed that the carotid artery stenosis had been improved by CAS (D). DSA: digital subtraction angiography

virtual histology intravascular ultrasound (IVUS) assessments, and the Precise stent (Cordis, Miami, FL, USA) or the Wall stent (Boston Scientific, Natick, MA, USA) systems were used. Hence, a Wall stent was placed when a plaque contained a rich necrotic core or if the plaque was soft, while a Precise stent was placed for other lesions. Pre-dilation (Fig. 1B) and post-dilation (Fig. 1C) was performed in all cases. For pre-dilatation, a balloon $4.0 \mathrm{~mm}$ in diameter was applied to calcified lesions, while a balloon $3.0 \mathrm{~mm}$ or $3.5 \mathrm{~mm}$ in diameter was applied to other lesions. For post-dilatation, a balloon $4.5 \mathrm{~mm}$ or $5.0 \mathrm{~mm}$ in diameter was applied to calcified lesions, while a balloon $4.0 \mathrm{~mm}$ in diameter was applied to other lesions. Several manual aspirations of the ICA blood within the ICA stump column were performed. After the CAS procedure, we confirmed the dilation of the carotid artery using angiography (Fig. 1D). Following CAS, argatroban therapy $(2.5 \mathrm{mg} / \mathrm{hr})$ continued for $12 \mathrm{~h}$. Dual antiplatelet therapy was administered for 3 months, and single antiplatelet therapy was prescribed indefinitely thereafter.

\section{Statistical analysis}

The differences between the treatment groups with respect to the continuous variables, namely, age and the degree of stenosis, were evaluated using Student's t-test. For the categorical variables, the chi-square test was used to determine differences between the groups with respect to sex, hypertension, hyperlipidemia, diabetes mellitus, smoking, ischemic heart disease (IHD), peripheral artery disease, and symptomatic stenosis, and Fisher's exact test was used to determine differences between the groups with respect to the perioperative complications P-value of $<0.05$ was considered statistically significant. All of the statistical analyses were conducted using SPSS software (IBM, Armonk, NY, USA).

\section{Results}

All patients were successfully treated and showed satisfactory dilatation. No significant differences were observed in the baseline characteristics except for the IHD of patients between the CEA and CAS group (Table 1). There were no MI, cardiac symptoms or renal failure following either procedure. In CEA group, there was one TIA and one major stroke due to acute occlusion of the ICA. In CAS group, there was one minor stroke and one major stroke (Table 2).

Minor and local complications in the CEA group comprised post-operative transient hypoglossal nerve palsy in 


\begin{tabular}{lccc} 
Table 1 Summary of clinical characteristics & \multicolumn{3}{l}{} \\
& CEA & CAS & p-value \\
Lesion & $104(46.8 \%)$ & $118(53.2 \%)$ & \\
Age: Mean \pm SD & $69.2 \pm 2.44$ & $72 \pm 3.43$ & $0.245^{* *}$ \\
Male & $93(89.4 \%)$ & $100(84.7 \%)$ & $0.347^{*}$ \\
Female & $11(10.6 \%)$ & $18(15.3 \%)$ & $0.313^{*}$ \\
Vascular risk factors (\%) & & \\
DM & $38(36.5 \%)$ & $46(38.9 \%)$ & $0.715^{*}$ \\
HT & $86(82.7 \%)$ & $108(91.5 \%)$ & $0.556^{*}$ \\
HL & $39(37.5 \%)$ & $48(40.7 \%)$ & $0.652^{*}$ \\
PAD & $35(33.6 \%)$ & $41(34.7 \%)$ & $0.543^{*}$ \\
IHD & $11(10.6 \%)$ & $30(25.4 \%)$ & 0.023 \\
Smoking & $75(72.1 \%)$ & $87(73.7 \%)$ & $0.835^{*}$ \\
Hyper-echoeic plaques on US & $44(44.4 \%)$ & $47(39.8 \%)$ & $0.632^{*}$ \\
Echogenic plaques on US & $40(38.5 \%)$ & $53(44.9 \%)$ & $0.568^{*}$ \\
Low-echoeic plaques on US & $20(19.2 \%)$ & $22(18.6 \%)$ & $0.551^{*}$ \\
Symptomatic cases & $55(52.9 \%)$ & $59(50.0 \%)$ & $0.694^{*}$ \\
Degree of stenosis (\% NASCET) : Mean \pm SD & $81.1 \pm 12.4$ & $82.5 \pm 13.2$ & $0.221^{* *}$ \\
\hline
\end{tabular}

No statistically significant by *Student's t-test, ${ }^{*}$ Chi-squared test. Statistically significant by Student's t-test shows bold type. CAS: carotid artery stenting; CEA: carotid endarterectomy; DM: diabetes mellitus; IHD: ischemic heart disease; NASCET: North American Symptomatic Carotid Endarterectomy Trial; PAD: peripheral artery disease; SD: standard deviation; HT: hypertension; HL: hyperlipidemia

\begin{tabular}{|c|c|c|c|}
\hline & CEA & CAS & $p$-value \\
\hline Lesion & $104(46.8 \%)$ & $118(53.2 \%)$ & \\
\hline HPS within 30 days & $1(0.9 \%)$ & $1(0.8 \%)$ & $0.524^{*}$ \\
\hline Minor stroke within 30 days & $1(0.9 \%)$ & $1(0.8 \%)$ & $0.524^{*}$ \\
\hline Major stroke within 30 days & $1(0.9 \%)$ & $1(0.8 \%)$ & $0.524^{*}$ \\
\hline Ml or death within 30 days & $0(0 \%)$ & $0(0 \%)$ & $1.000^{*}$ \\
\hline
\end{tabular}

*No statistically significant by Fisher's exact test. CAS: carotid artery stenting; CEA: carotid endarterectomy; HPS: hyperperfusion syndrome; Ml: myocardial infarction

one patient $(0.9 \%)$ and transient subcutaneous hematomas in three patients $(2.9 \%)$. Minor and local complications in the CAS group comprised new post-operative hyper-intense lesions on diffusion weighted images (DWI) in $21.8 \%$ of the patients, and subcutaneous hematomas associated with transient pseudo-aneurysms in two patients $(1.7 \%)$. Acute renal failure was not observed in our study. No significant differences were observed between the CEA and the CAS groups with respect to the perioperative complications that occurred within 30 days of the procedures. Total ratio incidence of perioperative complications, except minor and local complications, were $1.8 \%$ in CEA and $1.6 \%$ in CAS. Hyper-perfusion syndrome was observed in $0.9 \%$ of the patients in the CEA group and in $0.8 \%$ of the patients in the CAS group postoperatively (Table 2).

\section{Discussion}

In this study, we compared the CEA and CAS groups in relation to the perioperative complications within 30 days of the procedures, namely, stroke, MI, and death. There were no significant differences between the CEA and CAS groups with respect to the perioperative complications. This finding suggests that our structured and tailored treatment strategy that is based on selecting appropriate individualized treatment approaches in accordance with the patients' risk factors rather than adhering to a single treatment approach, will likely contribute to the attainment of favorable outcomes in all patients with carotid artery stenosis. At our institute, CAS is mainly performed for high-risk CEA cases, and the clinical outcomes from CAS are as good as those from CEA.

This study and a previously reported study ${ }^{17)}$ support the notion of CEA being the first choice of treatment and CAS being reserved for strictly selected cases, including those with re-stenosis after a previous carotid artery procedure, carotid artery dissection, ICA stenosis after radiotherapy, previous major neck surgery, contralateral cranial nerve palsy or tandem stenosis. In addition, CAS should be performed carefully in patients who are considered high-risk for both CEA and CAS. 
We confirmed that there were no significant differences between CEA and CAS with respect to the perioperative complications within 30 days of the procedures, and we observed low levels of perioperative complications. Especially, as for no occurrence of cardiac complications, our pre-operative cardiac assessments may contribute to such a good results.

A tailored CAS regimen requires a non-invasive diagnostic work-up that includes plaque-imaging using ultrasonography, CT angiography, and to select the most appropriate embolic protection device, for example, one that provides proximalflow blockage/reversal in the carotid artery, a distal-filter, or an occlusive balloon and stent type, namely, closed-cell or open-cell types. ${ }^{10)}$ Our CAS procedure utilizes proximal blockage and distal-filter protection and the stent types used vary depending on the virtual histology findings derived from the IVUS assessments. The outcomes of our procedure are better as the same as the tailored CAS reported by Miyachi et al. ${ }^{10)}$

Performance and results of CAS depends on progress in device development. ${ }^{8,14,18)}$ The Angioguard XP distal protection filter was replaced by the FilterWire EZ and Spider distal protection filters, which are more effective than at preventing distal embolisms and the latter devices have been approved for reimbursement from the National Health Insurance system. ${ }^{18)}$ Although remarkable advances have been made in relation to CAS devices, it is important for operators to be familiar with the devices and to understand their performance before use, so we choose proximal protection that is provided by a $9 \mathrm{Fr}$ OPTIMO balloon guiding catheter, and distal filter protection that is provided by a Spider embolic protection device.

There is general consensus that protection should be performed to prevent ischemic complications associated with CAS, and there are two types of protection devices, namely, filter and balloon, and two protection strategies, namely distal and proximal. Filter protection systems are widely available and are easily maneuverable, and they allow the antegrade carotid artery flow to be maintained during the procedure. However, the filter itself may allow the passage of some debris. Compared with distal balloon occlusion, filters have some disadvantages; including the passage of debris through or along the filter, for example, they have to allow particles that are smaller than their pore sizes to pass through them or, if the filter is not appropriately fitted to the vessel wall, they have to allow particles to pass along the filter. A proximal protection system is considered to be more effective at preventing embolization, mainly because it is placed and functioning before the distal protection device crosses the stenotic lesion and because it induces reversed flow by occluding both the external carotid artery (ECA) and the common carotid artery (CCA). A previous study ${ }^{16)}$ compared 50 CAS procedures using distal filters with $31 \mathrm{CAS}$ procedures that used a combination of proximal occlusion of the CCA only without ECA occlusion and a distal filter. The findings from this study led the authors to conclude that combining the use of a distal filter with proximal protection reduced the amount of debris captured by the distal filter and the occurrence of post-procedural ischemic lesions observed on DWI. Dual protection, which is provided by using proximal occlusion and a distal filter during CAS, seems to be a very protective and promising approach to the prevention of distal embolization.

In recent years, the outcomes from patients administered medical treatment for carotid artery stenosis have also improved. ${ }^{13)}$ In this study, two of the patients who developed complications following CAS were considered to be high-risk patients for both CEA and CAS. At present, for high-risk cases, there is no evidence to suggest that the treatment outcomes from CEA and CAS are superior to those from the best medical treatment. In Japan, where the incidence of natural ischemic vascular events is low, ${ }^{9}$ medical treatment may also be considered for patients who appear to be high-risk for both CEA and CAS.

There might be some limitations to this study, which includes lack of information regarding the risks associated with CAS, for example, vulnerable plaques or severe aortic valve stenosis. Further studies are required to determine the safety and efficacy of our treatment approach to the treatment of carotid artery stenosis.

\section{Conclusion}

Selecting appropriate individualized treatment methods based on patients' risk factors may contribute to improvements in the overall outcomes in patients with carotid artery stenoses. To achieve good outcomes, high-risk CEA patients should be treated using CAS with proximal protection and distal filter protection. Structured and tailored treatment for carotid artery stenosis led to good clinical outcomes, and CAS attained the same clinical outcomes as CEA, even in patients with lesions that presented a high risk for CEA.

\section{Acknowledgements}

The institutional review board approved this study, and the requirement for informed consent was wavied. 


\section{Conflicts of Interest Disclosure}

I certify that there is no actual or potential conflict of interest in relation to this article. The authors have no personal, financial, or institutional interest in any of the drugs, materials, or devices used in the study and described in this article.

\section{References}

1) Shinohara Y, Ogawa A, Suzuki N, et al. The joint committee on guidelines for the management of stroke. In: Japanese Guidelines for the Management of Stroke, Kyowa-Kikaku, Tokyo 2009, 123-4 (Japanese).

2) Brott TG, Hobson RW, Howard G, et al.: CREST Investigators. Stenting versus endarterectomy for treatment of carotidartery stenosis. N Engl J Med 2010; 363: 11-23.

3) Economopoulos KP, Sergentanis TN, Tsivgoulis G, et al. Carotid artery stenting versus carotid endarterectomy: a comprehensive meta-analysis of short-term and long-term outcomes. Stroke 2011; 42: 687-92.

4) Ederle J, Dobson J, Featherstone RL, et al.: International Carotid Stenting Study investigators. Carotid artery stenting compared with endarterectomy in patients with symptomatic carotid stenosis (International Carotid Stenting Study): an interim analysis of a randomised controlled trial. Lancet 2010; 375: 985-97.

5) Silver FL, Mackey A, Clark WM, et al.: CREST Investigators. Safety of stenting and endarterectomy by symptomatic status in the Carotid Revascularization Endarterectomy Versus Stenting Trial (CREST). Stroke 2011; 42: 675-80.

6) Yadav JS, Wholey MH, Kuntz RE, et al.: Stenting and Angioplasty with Protection in Patients at High Risk for Endarterectomy Investigators. Protected carotid-artery stenting versus endarterectomy in high-risk patients. N Engl J Med 2004; 351: 1493-501.

7) Marmot MG, Syme SL, Kagan A, et al. Epidemiologic studies of coronary heart disease and stroke in Japanese men living in Japan, Hawaii and California: prevalence of coronary and hypertensive heart disease and associated risk factors. Am J Epidemiol 1975; 102: 514-25.

8) Robertson TL, Kato H, Rhoads GG, et al. Epidemiologic studies of coronary heart disease and stroke in Japanese men living in Japan, Hawaii and California. Incidence of myocardial infarction and death from coronary heart disease. Am J Cardiol 1977; 39: 239-43.

9) Steg PG, Bhatt DL, Wilson PW, et al.: REACH Registry Investigators. One-year cardiovascular event rates in outpatients with atherothrombosis. JAMA 2007; 297: 1197-206.

10) Miyachi S, Taki W, Sakai N, et al.: Japanese CAS Survey Investigators. Historical perspective of carotid artery stenting in Japan: analysis of 8,092 cases in The Japanese CAS survey. Acta Neurochir (Wien). 2012; 154: 2127-37.

11) Marquardt L, Geraghty OC, Mehta Z, et al. Low risk of ipsilateral stroke in patients with asymptomatic carotid stenosis on best medical treatment: a prospective, population-based study. Stroke 2010; 41: e11-7.

12) Siewiorek GM, Wholey MH, Finol EA. In vitro performance assessment of distal protection devices for carotid artery stenting: effect of physiological anatomy on vascular resistance. J Endovasc Ther 2007; 14: 712-24.

13) Spence JD, Coates V, Li H, et al. Effects of intensive medical therapy on microemboli and cardiovascular risk in asymptomatic carotid stenosis. Arch Neurol 2010; 67: 180-6.

14) Gray WA, Hopkins LN, Yadav S, et al::ARVHeR Trial Collaborators. Protected carotid stenting in high-surgical-risk patients: the ARCHeR results. J Vasc Surg 2006; 44: 258-68.

15) Iyer SS1, White CJ, Hopkins LN, et al.: BEACH investigators. Carotid artery revascularization in high-surgical-risk patients using the Carotid WALLSTENT and FilterWire EX/ EZ: 1-year outcomes in the BEACH Pivotal Group. J Am Coll Cardiol 2008; 51: 427-34.

16) Harada K, Morioka J, Higa T, et al. Significance of combining distal filter protection and a guiding catheter with temporary balloon occlusion for carotid artery stenting: clinical results and evaluation of debris capture. Ann Vasc Surg 2012; 26: 929-36.

17) Bradac O, Mohapl M, Kramar F, et al. Carotid endarterectomy and carotid artery stenting: changing paradigm during 10 years in a high-volume centre. Acta Neurochir (Wien) 2014; 156: 1705-12.

18) Siewiorek GM, Wholey MH, Finol EA. In vitro performance assessment of distal protection devices for carotid artery stenting: effect of physiological anatomy on vascular resistance. J Endovasc Ther 2007; 14: 712-24. 